\title{
Service Robots for Special Education of Children with Disabilities
}

\author{
Robotized Systems for Social Applications
}

\author{
Roman Zahariev $\dagger$ \\ Institute of Robotics, \\ Bulgarian Academy of Sciences, \\ Sofia, Bulgaria \\ roman.zahariev@abv.bg
}

\author{
Nina Valchkova \\ Institute of Robotics, \\ Bulgarian Academy of Sciences, \\ Sofia, Bulgaria \\ nvalchkova@abv.bg
}

\author{
Hiroaki Wagatsuma \\ Kyushu Institute of Technology \\ (KYUTECH) \\ Kitakyushu 808-0196, JAPAN \\ waga@,brain.kyutech.ac.jp
}

\begin{abstract}
The article presents a methodology for using service robots in the process of rehabilitation for children in special education. The aim of the study is to provide effective experimental use of an innovative model for overcoming the social exclusion of disabled people (with special focus on young people), ensuring a dignified and independent life by piloting an intelligent modular service mobile robots called "AnRI" (Anthropomorphic Robot Intelligent). The EC-Funded Marie Curie Project H2020-MSCARISE-2017, No77720, Cyber-Physical Systems for Pedagogical Rehabilitation in Special Education, (CybSPEED), is contributing to the introduction of a model for the use of robotic technologies and "AnRI" robots in special education by disabled children and young people. The opportunity to explore experience and sustainable cooperation between beneficiaries and partners was used in the frame of the Project. It is shown, using from Service Robots "AnRI", the innovative approach, developed by KyuTech University - Japan, a partner in the CybSPEED project, for transmitting information with the help of "Key Words". The generation of knowledge and expertise in the field of pedagogical rehabilitation of people with disabilities is described, which will help to identify the needs of the target group of students with the use of service robots. It is shown also the processes of the study, testing of robotic assistive devices for people with disabilities and knowledge sharing, which provides project partners with completely new tools and forms of assistance for people with disabilities and will contribute to the improvement of existing practices. In the methodology described, not only an innovative element, the use of "AnRI" robots, but also new solutions for social problems, which will implemented in good cooperation with the professional team of the Social Services Center "St. Nedelya", Sandanski, Bulgaria.
\end{abstract}

\section{CCS CONCEPTS}

Hardware $\rightarrow$ Communication hardware, interfaces and storage $\rightarrow$ Sensors and actuators

$\dagger$ Corresponding author

Permission to make digital or hard copies of part or all of this work for personal or classroom use is granted without fee provided that copies are not made or distributed for profit or commercial advantage and that copies bear this notice and the full citation on the first page. Copyrights for third-party components of this work must be honored. For all other uses, contact the owner/author(s).

CompSysTech '20, June 19-20, 2020, Ruse, Bulgaria

(C) 2020 Copyright is held by the owner/author(s).

ACM ISBN 978-1-4503-7768-3/20/06. https://doi.org/10.1145/3407982.3408023
Hardware $\rightarrow$ Communication hardware, interfaces and storage $\rightarrow$ Electro-mechanical devices

\section{KEYWORDS}

Service robots, Robots control, User interface, Serving disadvantaged people

\section{ACM Reference format:}

Roman Zahariev, Nina Valchkova, Hiroaki Wagatsuma. 2020. Service Robots for Special Education of Children with Disabilities: Robotized Systems for Social Application. In Proceedings of $20^{\text {th }}$ International Conference on Computer Systems and Technologies (CompSysTech'2020). ACM, New York, NY, USA, 7 pages. https://doi.org/10.1145/3407982.3408023

\section{INTRODUCTION}

Problems in social communication accompany a number of disorders such as childhood cerebral palsy, autism, epilepsy, genetic diseases, hyperactivity and other undiagnosed conditions in childhood. An important task of pedagogical rehabilitation is to overcome the communicative barriers to the acquisition of knowledge and skills taught by the teacher and parents. Teaching aids make it more fun for children and more frugal to the resources of attention and concentration in learning. Technological "toys" are an invaluable aid in overcoming a number of symptoms - motor, perceptual and cognitive. Recent research results show, on the one hand, the wide range of disorders and, on the other, the dependence on a lack of motivational or cognitive resources rather than a genetic predisposition to disease. These results open up great prospects for rehabilitation robotics, and in particular for the use of robots to facilitate the pedagogical process by fulfilling the role of technological assistant to the teacher.

The task is to develop a laboratory model of a cognitive service robot with a manipulator as follows:

- The mobile robot is equipped with redundant anthropomorphic manipulation structure - a mechanical arm with a wrist of 3 fingers.

- The mobile robot must be equipped with the technical vision and the necessary sensors to ensure non-collision movement in a stochastic unordered environment and object recognition in it. The features that the robot should have are: 
- Achieving desired stereotyped mechanical arm configurations, "gestures".

- It is possible to realize Pick-up operations with a wide range of everyday items for children.

- Recognizing hand gestures of people in real space and time [15]. The robot's work environment should be close to that of the child. The robot should respond in a controlled manner to a wide range of signals emitted by non-homogeneous sources - sensors, humans, information protocols, etc. The reactions must sufficiently guarantee the safety of the child and the people in the movement area. the robot, as well as the safety of the robot itself from the aggressive impulses of the children (as far as possible).

\section{The Relevance of the Problem}

The study of literature, as well as the experience of experts with children with special educational needs, shows that it is extremely important to introduce into work with such children a robot assistant who can manipulate objects and show gestures, as well as recognize gestures displayed by person. In this way, existing practices can be significantly improved as well as new practices in dealing with children with special educational needs can be created.

The Assistant Robot provides the educator with the opportunity to manage communication processes, to gain knowledge of these processes, to perform analysis and to generate new approaches in their practice.

On the other hand, practice shows that a child is more likely to trust communication with the robot, since the robot is completely predictable, its reactions can be the same on the same stimuli. The robot is free of redundant information and can customize to work with each child individually.

Preliminary studies show that there is a great need for pedagogical rehabilitation-assisted robots that are capable of manipulating children's everyday items, that is, to carry out pick-and-place operations.

According to expert educators, the exchange of objects is an essential part of the communication process. Such communication between the child and the outside world greatly increases the effectiveness of the pedagogical process [6].

The project is developing such an Anthropomorphic Robot with Intelligent is robot with capabilities for manipulating objects, displaying gestures and recognizing gestures in real time. The development of the assistant robot was carried out jointly with specialists who actually practice the pedagogical rehabilitation of children with special educational needs .

With the discovery of the system of "mirror neurons" in the brain of primates, the theory of "imitation learning" in pedagogical rehabilitation began to develop intensively. "Mirror neurons" respond to an observed action in the same way as performing this action. The system of such neurons has the functional role of combining one's own senses with the perception of similar sensations in other people. A number of researchers have posited this system as the neurological basis of empathy - a sense of empathy with the emotions of others.
The theoretical hypothesis set out in this project is based on the theory of "M.D.'s empathy" from Lieberman [10]. According to this theory, empathy as subjective feeling can be of two types empathic attitude and empathic power. The hypothesis formulated in the project places the empathic force at the center of the empathic relationship. Empathy is represented as a dimension, an innate characteristic of a person's personality.

What is new in the proposed concept of pedagogical rehabilitation with the help of robots assistants? It is the idea that empathy as a personality dimension builds from a complete lack of empathic feeling, most often manifested in behavior as a reaction to opposition, through imitation, which is the effort to resist that is, the alien emotional state, to the foreseeing, the antithesis of the other person's condition. The focus of attention is determined by the available cognitive resource. The following theoretical hypotheses were tested:

- In the case of hyperactivity and attention deficit, the area of focus is too wide. Attention is superficial.

- In conditions such as autism or epilepsy, the focus of attention is narrowed and shifted away from the center. These hypotheses were tested in a study in which robots displayed autonomous behavior.

\subsection{Pedagogical Problems Solved through the Service Robots - Assistants}

The Robots from series "AnRI" (Anthropomorphic Robot Intelligent) was developed, in the Institute of Robotics at Bulgarian Academy of Sciences like Service Robots. As is well known Pedagogical Rehabilitation Toys for Robotics are currently being developed intensively not only for children and not only for dealing with muscle hypertension, although they are the most common.

The new ideas in the proposed pedagogical robotics management system is that it is based on a "Know-How" methodology for teaching gestures to children with communicative problems, developed based on the experience of the Social Services Center "St. Nedelya", Sandanski, Bulgaria. The Robot will assist the teacher in the training experiment and will demonstrate the learning of child through imitation. New concrete indicators of the child's progress in communication skills training will propose.

The use of Information Technology in pedagogy is currently at its peak because of the great technological capabilities of the environment in which modern children grow up. The systems for analyzing social situations, for analyzing non-verbal behavior, for recognizing gestures, etc., are not yet sufficiently precise because of the complexity of the task of identifying a human body and a moving object at the same time [12].

This project applies an original team approach that states that the social competence of an information system for recognizing people in social situations is achieved when the information system is capable of recognizing an action as a result of an attitude, not just as a result of another action (such as physical object). The specific pedagogical problems that the project addresses directly by creating a robot assistant are the following: 
1. The need to attract the attention of the child, e.g. if he sees that the therapist manages to get the robot to open his hand through gestures, he will try. This is useful for children with cerebral palsy or impaired mobility.

2. Children with autism, for example, are known to prefer to communicate with a technician rather than a therapist. In other children, the factor is boredom by the therapist. In the particular problem area, the lack of emotions in the robot is a positive factor that brings predictability and security from a child's perspective. Tactile contact will facilitated, which is important for these children.

3. The shortening of training time through a team of therapist and assistant robot is the expected result after the implementation of the project. The main goal is the technological development of pedagogical rehabilitation, so more attention is paid to testing the device itself and its acceptance by children, than to testing strict statistical hypotheses.

\subsection{Requirements for the Functional Characteristics of Robots "AnRI"}

The purpose of this activity is to investigate the extent to which the developed robot contributes to ensuring a decent and independent life (with a focus of youth) on people with disabilities. The functional characteristics of the robot will be analyzed in terms of objectives set for serving disadvantaged people. Here are some specific features, such as planning for a safe trajectory of motion in the context of the dynamically changing environment in which the serviced person lives. Also, the use of sensor information from "infrared" sensors and "ultrasonic" sensors, as well as "End-Off" sensors built into the robot, to prevent collisions in the work environment. Ability to build databases with the inclusion of libraries from readymade programs to perform standard commands such as "Bring me the medicines!", "Bring me the milk!" and others are made. During the experimental use of the robot, a report will be prepared with the results of the robot application as a lecturer in the special education.

\subsection{Reliability Requirements for Robots "AnRI"}

$>$ Robot reliability requirements are increased relative to standard requirements for man-machine interface for the following reasons:

- Work in close proximity to the serviced person.

$>$ - Responsibility for the accomplishment of the assigned tasks, for which there is no alternative solution and the failed implementation, can lead to unpredictable and tragic consequences.

> Serving disadvantaged people is extremely important to carry out reliable equipment, because otherwise there may be significant mental injuries.

$>$ The unprofitable work of the robot can also lead to specific disabilities in contact with the serviced person.

The project has also developed an Attestation Card, in which will be made suggestions from the pedagogical specialists in the experiments with the people from the target group and so they will be able to express their opinion regarding the use of the "AnRI" robots for servicing the people in unequal position.

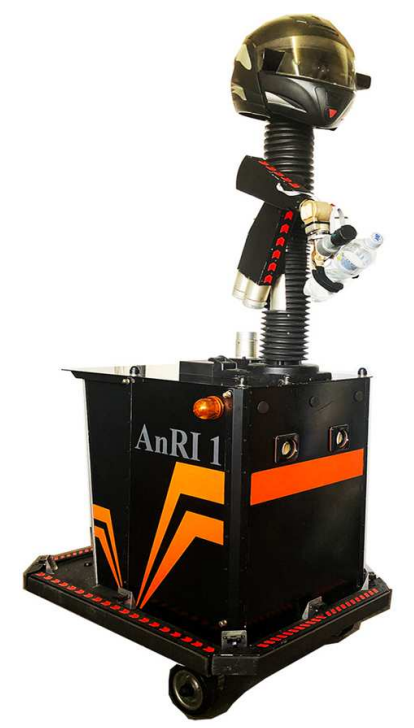

Figure 1: View of the Service Robot "AnRI-1"

\section{Description of the Created Robots "AnRI-1" \& "AnRI-2"}

The Robots "AnRI-1" and "AnRI-2" (Fig. 1 and Fig. 2) are realized on the base of constructions of mobile platforms with four wheels, which two are driven and two are independent "free" wheels. The wheels in the Robot "AnRI-2" are located in the form of cross. The "free" wheels are at the rear side one and in front side the other of the platform. The driven wheels are at two sides. With the help of this construction of the platform, it is possible to control the Robots movements around the vertical axis around geometrical center of construction in the left and right sides [18]. Electric motors, DC powered by a rechargeable battery are built at the hub of the driven wheels. "Worm" gearboxes that do not allow movement back using their braking effect, are used in the driven wheels of the Robot platform.

The same construction has the Robot "AnRI -1" with different position of the wheels, which are mounted in the form of "triangle". In front of the two tops of the triangle are located the driven wheels and at the third top in the rear side is two "free" wheels.

The manipulator of anthropomorphic type with three regional and three local degrees of mobility and gripper with separate drives and with three fingers, is mounted at the top of the robot "AnRI1 " $[16,17]$.

\subsection{Drive and Technical Characteristics of the Robot Manipulator}

Based on "servo" controllers with feedback from incremental sensors, located in each degree of mobility, the drive of the Robot 
Manipulator is realized. All regional joints are equipped with electromagnetic brakes [9].

Technical characteristics of the Robot Manipulator are as follows:

$>$ Coordinate system type is Anthropomorphic type.

$>$ Structure: 3 regional and 3 local rotational degrees of mobility + driven by the gripper.

$>$ Structure formula is RRR / RRR + Gr.

$>$ Positioning accuracy is $+-0.05 \mathrm{~mm}$.

$>$ Repeatability positioning accuracy is $+0.05 \mathrm{~mm}$.

$>$ Maximum travel speed of the manipulator is $0.3 \mathrm{~m} / \mathrm{sec}$.

$>$ Maximum travel speed of the robot is $0.5 \mathrm{~m} / \mathrm{sec}$.

$>$ Power: Rechargeable $12 \mathrm{~V}$. (Rechargeable battery required).

$>$ Weight of the manipulator is $6.75 \mathrm{~kg}$.

$>$ Working area dimensions in horizontal plane are $1 \mathrm{~m} \times 1 \mathrm{~m}$.

$>$ Loading capacity is $1.5 \mathrm{~kg}$.

$>$ Motor drives are - Electric - DC Servo. It is used Robot Operating System (ROS)

$>$ Both at regional and local degrees of mobility of Manipulator it is possible to control the speed of execution.

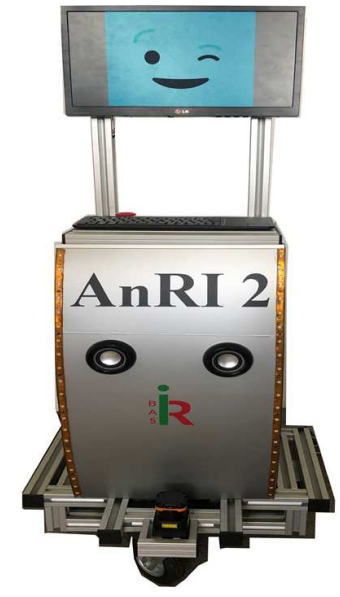

Figure 2: View of the Service Robot "AnRI-2"

\subsection{The Control System of the Robots "AnRI"}

Hierarchical, distributed and microprocessor type is The Control System of The Robots. It is consist from different levels and is realizes control of different devices and systems. It is possible used corresponding software modules [1,5]. The connection between all devices on the management is realized via serial interface RS 232. CPU module for total robot control is based on 32-bit microprocessor unit. In the recent years 32-bit microcontrollers are widely used for robot control applications. Most popular and wide spread architecture is Cortex-M processor family [16]. In our opinion, the Cortex-M4 family is well suited for robot control applications. It is integrates Digital Signal Processing (DSP) unit with processing of floating-point support for fast and a power-efficient algorithm. Therefore, Cortex-M4 can use in digital control applications such as motor control, sensor fusion and power management [11].
In this paper was described a practical realization of Cortex-M4 architectural Control System based on universal I/O board for Service Robots. On the same base is realized control a set of digital and analog inputs, 10/100 Ethernet interface, PWM controls and used CAN, SPI and I2C buses [2, 3 and 4]. This configuration enables flexible solution for connecting various sensors and actuators of the robots. In the same time using, the modern Cortex-M4 architecture allows simplify the design and reduce amount of external components. This greatly improves the whole system reliability [16]. Figure 3 shows a block diagram of the developed communication concept for "AnRI" robot control. The use of Mixed Reality Server Node plays a special role. This component provides combined information from the mapping server, the navigation component, and the robot's knowledge base for environmental objects. The information is sent as a stream to create augmented reality to the User Interface with Graphical User Interface (GUI). It performs part of the data processing before sending it to the User Interface [1].

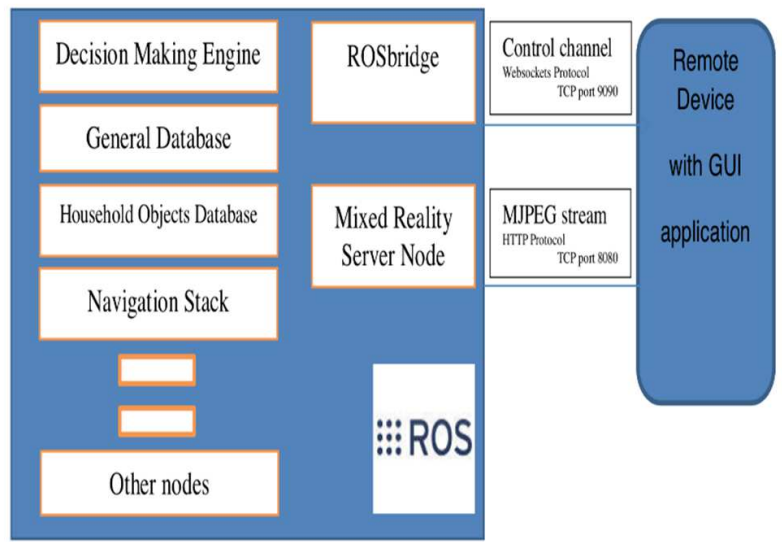

Figure 3: Block Scheme of the developed communication concept of The Robots "AnRI"

This is to optimize the use of the network bandwidth, to realize synchronous access to information from different sources, and to reduce the computational requirements for the device that implements the GUI. Using a standard control TCP/IP [4] network for communication with the board allows platform independent control.

Also various diagnostic tools and standard libraries can be used for rapid implementation. It is very important, is presented another serial interface modules, GPS module for navigation and GPRS for Internet communication [14].

This is allows to be used of vision module and the module of the laser interferometer, along with integration module, reading and loading data from sensor systems. Used operating system is ROS (Robot Operating System). [7, 16].

\section{Functional Experiments and Possible Scenarios with the Robots "AnRI"}

Functional Experiments and possible test scenarios can be considered and realized together with specialist pedagogues as 
well as psychologist, who will assist in the trials and who care for the person being served [8]. In the Experiment, the trainee will be sitting at a table with a back to the camcorder that documents the experiments. As the initial adaptation of the learner to the robot, the following series of experiments carried out: For example, a glass of liquid, water or milk can delivered to the attendant. The action involves bringing a cup and leaving it on the table in front of the service (Fig.5). Then the robot brings a bottle of liquid and pours it into the glass. Finally, the robot takes the bottle and puts it in the refrigerator, and the empty glass goes to the washbasin. Another possible scenario is the Robot bring of a newspaper or book from the bookshelf of the library. The action involves from the bookshelf of the library Robot bringing the newspaper or book and placing it on the table.

\subsection{The Evaluation for the Communication between the Robot and the Learner}

The trained child is sitting on a table with a back to the camera that documents the experiments. In the same time during the experiment in the laboratory are Teacher Pedagogue, Psychologist, Researcher Programmer and Scientific Supervisor. It is very important from point of view of security: the table is between robot and the trained child. In front of the table there are geometric figures in the form of objects: for example a rubber ball with a diameter of $100 \mathrm{~mm}$, a dice made of cubic plastic material with dimensions of $100 \times 100 \times 100 \mathrm{~mm}$ and a canvas child's hat (Fig.4).

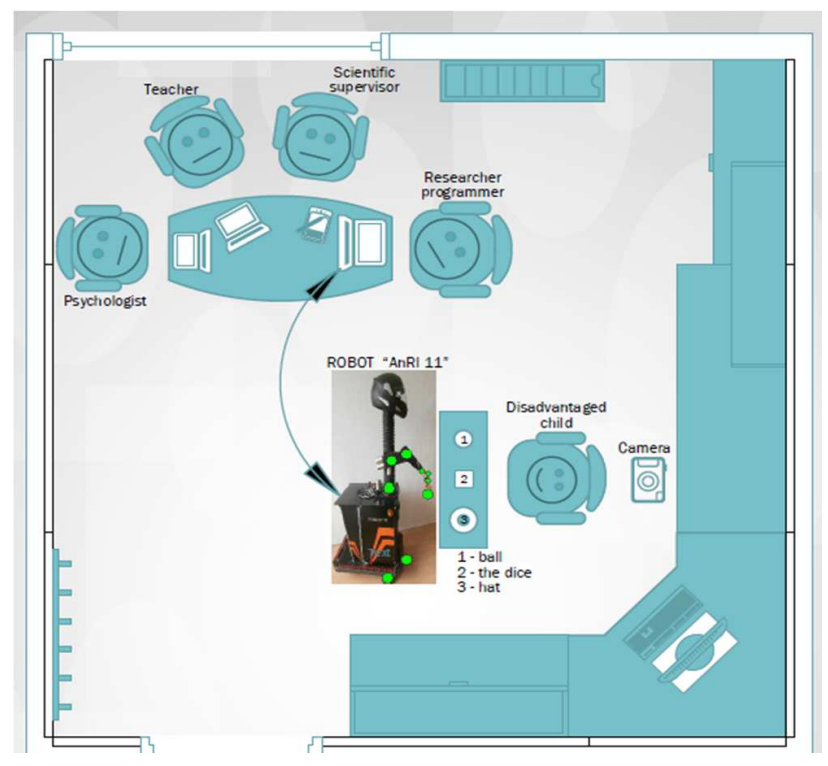

Figure 4: Scheme of the laboratory for the experiments with the help of service robot "AnRI-1"

Another Scenario is the trainee is sitting on a table with a back to the camera that documents the experiments. In front of him is the robot standing ready to start the experiment session. At the beginning, short robot commands are given to the learner to identify a particular subject by lifting it and then leaving it in place. If the answer is correct, the trainee gets a card from Set of playing cards for a reward. The number of cards received is the percentage of the student's correct answers. Later, the task is complicated, as the robot, for example, tells a short story and invites the learner to answer questions about history. For example, who is the "bad" character and who is the "good" character? How the good will beat the "bad forces" and deals with all the obstacles to victory. How the "bad" hero will fail in his vicious plan to threaten the "good" hero and how finally everything will end happily. It is imperative that the fairy tale has an optimistic sound and that it comes to some common human and humane conclusions. If a positive answer is recorded, the trainee receives a card from Set of playing cards for a reward.

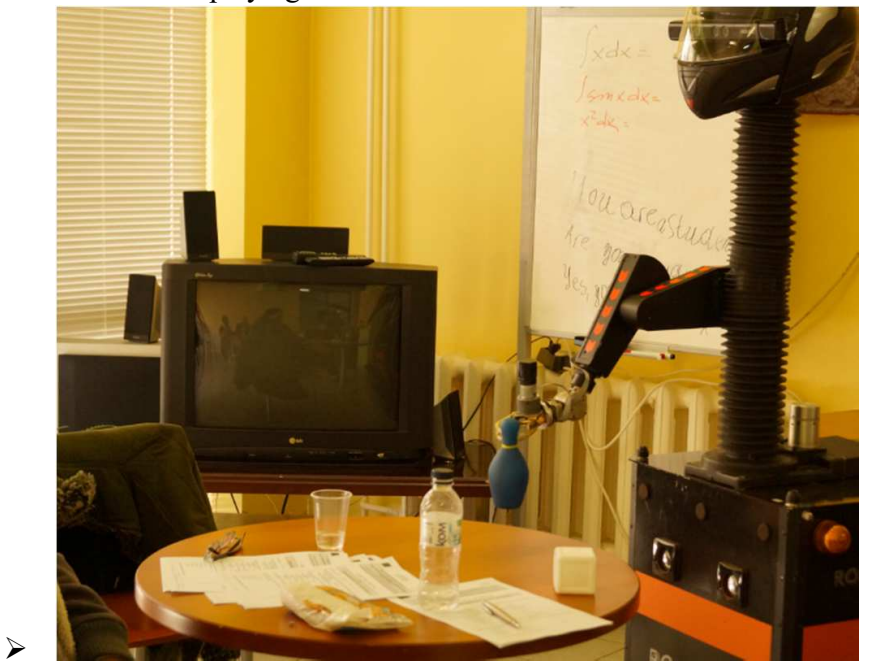

Figure 5: Layout of the laboratory for the experiments with the help of service robot "AnRI-1"

Another such scenario is the robot performs a short children's song by instructing the individual in the chorus to clap his hands in tact with the song. If successful, the student receives a card from Set of playing cards for a reward.

First, the robot performs the song independently. He then gives instructions on which places Lerner should clap his hands and how many times.

After the student's positive coaching experience, he receives a card prom Set of playing cards for a reward. Finally, the robot instructs the learner to clap his hands in the rhythm of the song during the whole performance. If attempt is successful, the student receives a card from Set of playing cards for a reward.

In the new Experiment, based on an innovative approach, developed in KyuTech University, Japan, a partner in the CybSPEED project, for transmitting information with the help of "Key Words" is possible to communicate with learner.

The "AnRI-2" robot will offer Learners a short text by highlighting the keywords in it. After that, Learner will try to retell the story with the help of the method of "key words". The 
Robot have an opportunity to assess degree of memorizing of the story from Learners and have a possibility to evaluate them on the base of comparing with the number of used "key words".

The number of received prize cards also takes into account the percentage of positive answers for scientists about the degree of learning by the learner of the transmitted information. Of course, such scenarios can compose together with the learner, depending on his/her specific requirements and wishes and with the help of the specialist pedagogue in the course of the experiments.

$>$ 3.2 Safety Measures for the Exercise of Experiments

The safety measures for handling the "AnRI" robots are extremely important. Since the robot is a type of device with a built-in human-machine interface, it is particularly important to master interface handling to prevent fatal errors from being committed.

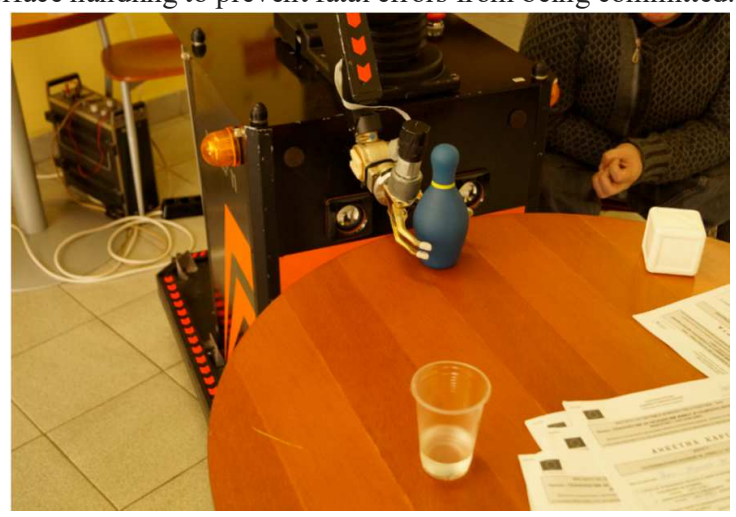

Figure 6: Service robot "AnRI-1" in process of shape recognition

$>$ Like any robot machine, you also need to consider the possibility of going out of the normal operating mode with all the unpleasant consequences of it. Therefore, it is recommended that the robot does not allow physical contact with the person being served and that a tangible agent, such as a table on which the objects to be handled and from which he takes service, is used. This will avoid direct contact between the robot and the trainee, which will guarantee the latter, is safety in their joint activities. Separately, a "stop" button is mounted in the top of the robot, which is in an accessible position on the robot platform, and it is possible at any time to be depressed, thus terminating the robot's operation.

\subsection{Test Results and Performing an Analysis of Learner Experiments}

Based on the video footage taken from the documentary camcorder and based on the completed protocols for the conducted experiments and the personal impressions of the participants, a detailed analysis of the factors determining the results of the experiments will carry out. The reasons behavior of the learners will analyzed and recommendations for improvement of the special education process will issued.

We will analyze the results of the transfer of the information to the trainees and the impact of the behavior of the "AnRI" robots on their consciousness based on statistical surveys of the percentage of positive responses received during the experiment. These results obtained with the presence of the robot in the process of special education will compared with those of the regular training of the trainees. On this basis, we will assess the effect of the application of robotic technology in the special education process and the possibilities for a certain therapeutic effect on the learners.

In the event of a camera facing the lens of the learners, the video will destroyed by creating a relevant protocol with the signatures of the Responsible Researcher and two of the Social Services Center. Such an experiment will consider invalid and will repeated.

\section{CONCLUSIONS}

The article shows the concept of information transmitted to the learner not only by verbal route but also by gestures and actions realized with the help of an AnRI-1 service robot. For this purpose, the use of a manipulator with a three-finger gripper in the robot, which allows to generate not only gestures, but also actions and manipulations in support of the information contact between the robot - the lecturer and the learner. Using the methodology described, one achieves an innovative effect in special training, which is very similar in nature to the way information is transmitted between people. The results of the laboratory tests proved to be very encouraging.

Young people with normal development were place in the role of trainees. A dialogue between the robot and the learner was observed, ending with a reported number of cards available for the positive responses received. The use of the AnRI-2 robot, which is equipped with a large screen for additional visualization and illustration of the ongoing dialogue, creates new opportunities in the process of transmitting information to the learner.

Based on the innovative approach developed by KyuTech University - Japan, a partner in the CybSPEED project, to convey information using "keywords", high communication efficiency with the learner is achieve and the ability to easily and quickly memorize key points from the ongoing dialogue. It should note that in this case, the elements of artificial intelligence in the robot - lecturer was use in the realization of the training, as long as the voice recognition of the received answers by the trainee is required.

When combining the two robots "AnRI-1" and "AnRI-2" simultaneously in the training and communication processes with the learner, it is possible to use the manipulator of the first robot and the capabilities of the large screen of the second robot to increase the efficiency of the dialogue. In this way, a cyber physical system of two robots is synchronously implemented, providing possibilities not only for verbal but also for gesture communication, which adds to the visualization of a large screen and voice recognition with elements of artificial intelligence.

In the framework of the CybSPEED project, pilot experiments will conducted in the territory of the Social Services Center "St. Nedelya", Sandanski, Bulgaria with the participation of trainees from the group of foster children at the Center and a team of 
pedagogical experts and psychologists. From educators, the children are also identifying as trainees and they are ready to carry out the first experiments in the Center.

After the first experiments, the results obtained will carefully analyzed using the service robot "AnRI-1", using the expertise of the readers of the Center. General conclusions and recommendations from the experiments and tests of the optimal behavior of the robot in the training conditions of disadvantaged people can done to improve the robot's work. Along with this, analyzes can also be made on observations, made about the robot's preferred behavior from the point of view of the serviced person. This will create new ideas for robot upgrading and its functional characteristics to better meet the specific requirements of people with disabilities.

Before proceeding with the implementation of the innovative method of training children with developmental problems in pedagogical practice, all the remarks and suggestions made by expert pedagogues will take into account in order to improve the algorithms used in the robot. The use of different scenarios and the preparation of specific lessons will generated and refined in the process of work with the help of experts - pedagogues and psychologists from the Social Services Center.

\section{ACKNOWLEDGEMENT}

The authors acknowledge the financial support of the European Union's Horizon 2020 Research and Innovation Program under the Marie Skłodowska - Curie Grant Agreement No 777720 (H2020-MSCA-RISE-2017 for project "Cyber-Physical Systems for Pedagogical Rehabilitation in Special Education" (CybSPEED).

\section{REFERENCES}

[1] Angelov, G., et al. (2011) „Remote Interface Communication to ROS Based Robotic System"; Proceedings of the Twenty First International Conference Robotics and Mechatronics; "Invited Session - Austrian-Bulgarian Automation Day"; 19-21 September 2011; Varna Bulgaria; ISSN 1310-3946; pp. $22-27$. https://www.acin.tuwien.ac.at/fileadmin/acin/ijaa/ijaajg20h2.pdf\#page $=22$

[2] Chih-Jer Lin, (2004) Motion Planning of Redundant Robots by Perturbation Method, An International Jurnal Mehatronics The Sciance of Intelligent Machines, Vol.14, No. 3, April 2004, pp.281-297. https://www.sciencedirect.com/science/article/abs/pii/S0957415803000321

[3] Chivarov N., Penkov Sv., Angelov G., Radev D., Shivarov N. and Vladimirov Vl., (2012), Mixed Reality Server and Remote Interface Communication for ROS Based Robotic System, International Journal Automation Austria (IJAA), ISSN 1562-2703, pp.144-155.

https://www.acin.tuwien.ac.at/fileadmin/acin/ijaa/ijaajg20h2.pdf\#page $=42$

[4] Chivarov N,, Paunski Y., Ivanova V., Vladimirov V., Angelov G., Radev D., Shivarov N., (2012), Intelligent modular service mobile robot controllable via internet, In: IFAC International Conference " SWIIS 2012", June 11th -13th, Waterford, Ireland, pp 149-153. https://doi.org/10.3182/20120611-3-IE4029.00029

[5] Cheah C.C., Y. Zhao, J. Slotine, (2006) Adaptive Jacobian motion and force tracking control for constrained robots with uncertainties, in: Proc. IEEE International Conference on Robotics and Automation, 2006, pp. 2226-2231. DOI: 10.1109/ROBOT.2006.1642034 https://ieexplore.iee.org/abstract/document/1642034

[6] Dimitrova M.,Vegt N., \& Barakova E. (2012) Designing a System of interactive robots for training collaborative skills to autistic children. Interactive Collaborative Learning ICL2012, Villah, Austria, IEEE, Xplore, pp. 1-8. DOI: 10.1109/ICL.2012.6402179

https://ieeexplore.ieee.org/abstract/document/6402179
[7] Doulgeri Z., Y. Karayiannidis, (2007) Force position control for a robot finger with a soft tip and kinematic uncertainties, Robotics and Autonomous Systems 55 (2007), pp. 328-336. https://doi.org/10.1016/j.robot.2006.11.003

[8] Kaburlasos, V.G., Dardani, Ch., Dimitrova, M., \& Amanatiadis, A. (2018). Multi-robot engagement in special education: a preliminary study in autism. Proceedings of the 36th IEEE International Conference on Consumer Electronic (ICCE), Las Vegas, USA, 12-15 January 2018, pp. 995-996. https://ieeexplore.iee.org/abstract/document/8326267

[9] Kurt S. Anderson, YuHung Hsu, (2002) Analytical Fully Recursive Sensitivity Analysis for Multibody Dynamics Chain Systems, Multibody Systems Dynamics., 08. 2002, Springer, ISSN: 1384-5640 (print) 1573-272X (online), pp. 1-27. https://link.springer.com/article/10.1023/A:1015867515213

[10] Liberman, R. P., DeRisi, W. J., \& Mueser, K. T. (1989). Social Skills Training for Psychiatric Patients. Pergamon Press. https://psycnet.apa.org/record/198998167-000 (PsycINFO Database Record (c) 2016 APA,)

[11] Paunski Y., R.Zahariev, (2017), Service robots control system, based on "Arm cortex M" architecture microprocessor system", Pr. TU Sofia, XXVI International Conference ,ADP-2017“", June, 2017, Sozopol, Bulgaria, pp. 300304. sofia.bg/MNTK\%20ADP\%20site/4\%20Proceeding/2017/Materials/Napravlenie -7/4-7-R.Zahariev-2.pdf

[12] Probst, R., P, Kopacek (1996), Service Robots: Present Situation and Future Trends, The Second ECPD International Conference on Advanced Robotics, Intelligent Automation and Active Systems, Vienna, Austria,1996, pp.45-52, https://www.sciencedirect.com/science/article/pii/S1474667017427376

[13] Serholt S., (2018), Breakdowns in children's interactions with a robotic tutor: A longitudinal study. Computers in Human Behavior, V.81,2018,pp.250-264. DOI: http://10.1016/j.chb.2017.12.090

[14] Khatib 0., P.Moore (1997), Sensor Based Control of Robots. Stanford University Publ, USA, 1997.

[15] Serpanos, D., (2018), The Cyber-Physical Systems Revolution. J. Computer, V.51(3), (2018), pp.70-73. DOI: https://doi.org/10.1109/MC2018.1731058

[16] Valchkova N., R. Zahariev (2019) Optimization of Model Operator for Service Robot, Intended to Service Persons with Disability. Pr. "TECIS-19" IFAC Conference on International Stability, Technology and Culture, 26-28 September, Sozopol, Bulgaria. https://doi.org/10.1016/j.ifacol.2019.12.468

[17] Zahariev R, N. Valchkova, G. Angelov, Y. Paunski, and A. Krastev. (2019), Robots for Help in Pedagogy and Rehabilitation. In Pr. 20-th International Conference on Computer Systems and Technologies (CompSysTech'19), ACM, New York, NY, USA, 1-7. DOI: https://doi.org/10.1145/3345252.3345253

[18] Zahariev R., N. Valchkova. (2019) Existing Robotics Technologies for implementation of Special Education. In book: M. Dimitrova \& H. Wagatsuma (Eds.) Cyber-Physical Systems for Social Applications, IGI-Global Pensylvania, USA, ISBN13: 9781522578796 DOI: http://doi.org10.4018/978-1-5225-7879-6 https://www.igi-global.com/book/cyber-physical-systems-socialapplications/210606 